\title{
Scendere in campo / mettere in campo
}

\author{
Mario Piotti
}

PUBBLICATO: 07 MAGGIO 2014

\section{Quesito:}

Rispondiamo a Fabrizio Gabrielli che, attraverso la nostra pagina Twitter, ci chiedeva un intervento sulle "metafore calcistiche" mettere in campo, scendere in campo.

\section{Scendere in campo / mettere in campo}

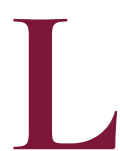

eggiamo dal "Corriere della Sera" del 22 febbraio di quest'anno: "Alla fine, il risultato lo si è visto. E Napolitano lo certifica come il frutto di quella "serena collaborazione istituzionale" descritta dall'articolo 92 della Costituzione. Ossia quelle scarne righe dove si precisa che "il presidente della Repubblica nomina, su proposta del presidente del Consiglio, i ministri". Ecco il nodo critico, sul quale si trascineranno le interpretazioni del futuro prossimo: chi ha prevalso su chi, posto che qualcuno abbia vinto sull'altro? O è meglio pensare che la partita, per stare al lessico calcistico ormai in uso a Montecitorio, si sia chiusa con un pareggio?" (p. 5)

L'autore dell'articolo, Marzio Breda, sembra volersi dissociare dalle proprie scelte linguistiche attribuendole al mondo della politica, delle cui parole il giornalista altro non sarebbe che resocontista. Una reazione infastidita sembra quella del giornalista nei confronti di parole e espressioni che pur da lui vengono usate ma dalle quali si distanzia, e delle quali mostra di non volere assumere il punto di vista. E le parole a cui si riferisce sono con tutta evidenza quelle relative all'ambito calcistico: partita e pareggio. Ė probabile però che nella diffusione di usi metaforici del linguaggio sportivo, e il calcio ne è la gran parte, viga nellitaliano contemporaneo un principio di corresponsabilità tra politica e giornali.

Ma facciamo un po' di storia. All'inizio degli anni Sessanta del Novecento, Carlo Bascetta poteva ancora scrivere che l'espansione del linguaggio sportivo nella lingua comune era molto limitata, e sottolineare che spesso si trattava di cavalli di ritorno, parole cioè che dalla lingua comune erano poi passate allo sport dove avevano assunto nuove accezioni, con le quali poi avevano intrapreso il cammino a ritroso verso la lingua comune. Ricordava in ogni caso, tra altre parole oggi in disuso, come debiti sportivi della lingua comune: pulcini, incassare, colpo basso, fuori giuoco, un successo ai punti, smarcarsi, rimanere al palo, mettere con le spalle a terra, mettere k.o., preso in contropiede, fuori tempo massimo, in zona Cesarini, seguire a ruota, limitandoli all'uso di "ambienti popolari". Tuttavia, già un decennio più tardi, Angelo Stella poteva ribaltare l'osservazione scrivendo di "conseguenze piuttosto perspicue"; e in particolare sottolineava da un lato il fatto che i prelievi avvenissero con vivacità dal linguaggio politico e dall'altro lato che uso e diffusione avvenissero per mezzo della stampa, con particolare addebito al quotidiano milanese "Il Giorno". Qualche esempio da sport diversi dal calcio: "i partiti si sentono al nastro di partenza di un notevole test elettorale" (I.5.7I), "si dispongono a ventaglio nella settimana conclusiva delle elezioni" (7.6.7I), "Galloni... aveva parlato di abbassamento di guardia democristiana di fronte al MSI" (I6.6.7I).

Più recentemente la pervasività del linguaggio sportivo in politica e nei giornali è stata pluralmente osservata dagli autori del volume collettivo Litaliano al voto, ampia disamina linguistica della campagna elettorale del 2006. Ancora Angelo Stella, nella Premessa, ribadiva l'impossibilità di "prescindere dalla parafrasi offerta dal sotteso palinsesto calcistico, affermatosi con "Il Giorno" degli 
anni Sessanta" (p. XXXI); e poi, dando la parola a uno per tutti dei giovani studiosi autori del volume, Edoardo Buroni osservava pacatamente:

[...] un favore significativo viene attribuito all'ambito sportivo; quest'ultimo consente molto spesso di presentare la stessa conflittualità che è possibile esprimere con metafore belliche ma in modo meno cruento e violento, senza per questo rinunciare alle componenti competitiva e contrappositiva tipiche anche dello sport. (p. I24)

Dunque, come si diceva inizialmente, vale la corresponsabilità: da un lato è la politica a servirsi dell'immagine sportiva, dall'altro fa comodo al giornalismo tradurre il confronto politico come confronto sportivo. E ciò appare del tutto evidente nel seguente esempio: non dettato dalle dichiarazioni del protagonista dell'articolo ne è infatti l'inizio: "Ẽ come giocare in casa, in undici contro dieci e con il vento a favore. Silvio Berlusconi scende in campo a Domenica live, accogliente salotto televisivo di Mediaset" ("La Repubblica", 28.04.20I4, p. II); qui nulla sottrae l'autore dell'articolo, Tommaso Ciriaco, dalla responsabilità delle proprie scelte linguistiche.

Si ritorna cosi, con esplicito compimento di ventennale visto il protagonista, all'impegno in politica di Silvio Berlusconi nel 1994, annunciato per l'appunto come una sportiva discesa in campo, evento che è poi divenuto consuetudine indicare come punto di riferimento per la diffusione dell'espressione sportiva nel linguaggio politico. L'imprenditore milanese annunciava infatti con ricchezza di metafore in specie calcistiche la propria decisione di dedicarsi all'attività politica, suscitando in molti l'impressione di andar oltre la metafora e di suggerire un'equivalenza tra politica e sport. Se dunque la politica diviene tale, ha bisogno di uno spazio dove esercitarsi, un campo, per l'appunto, dove scendere o dove mettere la propria squadra: dal punto di vista della lingua ciò parrebbe aver comportato un incremento d'uso di tali immagini, e certo ne ha comportato una loro maggiore visibilità. Proprio con riferimento agli usi linguistici berlusconiani, Gian Luigi Beccaria ne faceva infatti notare il compiacimento per le metafore calcistiche, tra le quali indicava scendere in campo, per altro espressione che poche pagine prima era stata segnalata come esempio di linguaggio militaresco spesso preso a prestito dalla politica (G.L. Beccaria, Per difesa e per amore. La lingua italiana oggi).

Ma disinteressandoci in questa sede delle vicende del politico lombardo, proviamo a seguire, pur in maniera del tutto desultoria, quelle delle due locuzioni verbali scendere in campo e mettere in campo servendoci dapprima del Grande dizionario italiano dell'uso di Tullio De Mauro (GRADIT). Per la prima delle due locuzioni il GRADIT dà questa definizione: "presentarsi a gareggiare, affrontare una competizione, misurarsi con qualcuno" e aggiunge come significato figurato: "assumere una posizione pubblica, un ruolo politico"; mentre per la seconda scrive: "far scendere in campo; schierare in un combattimento o in una gara: $\mathrm{m}$. in campo tutte le truppe, m. in campo la squadra al completo"; anche di questa dà un significato figurato: "addurre, presentare: ha messo in campo inutili scuse e pretesti". Né dell'una né dell'altra indica la data di prima attestazione (ma è così per quasi tutte le locuzioni polirematiche).

Il Grande dizionario della lingua italiana di Salvatore Battaglia (GDLI) ci informa che campo può essere, tra altri significati, nell'ordine: lo spazio dedicato ai duelli, ai tornei, il luogo della battaglia, può indicare figuratamente la contesa, la lotta, il contrasto, la discussione; tra gli ultimi significati indica anche quello di 'terreno su cui si svolgono le gare sportive', per il quale, tralasciando un esempio di Gabriello Chiabrera forse non del tutto pertinente, adduce solo esempi novecenteschi. Per mettere in campo [s.v. campo] indica i seguenti significati: 'addestrare e assistere il cavaliere durante la giostra', con un primo esempio cinquecentesco da B. Varchi; 'far combattere come proprio campione', con primo esempio da Andrea da Barberino; 'schierare in combattimento, mobilitare per la guerra', con primo esempio dal volgarizzamento del Milione di Marco Polo; 'addurre argomenti', il primo esempio è dalla 
Clizia di Machiavelli. Non ne dà il significato sportivo. Non è privo di significato che sempre il GDLI [s.v. scendere] documenti scendere in campo nel significato di 'schierarsi per gareggiare' solo a partire dal 195I, e lo faccia con un esempio tratto dalle Noterelle calcistiche di Lanfranco Caretti, apparse su "Lingua Nostra" in quell'anno. Mentre sempre dal GDLI si ricava che, dei tre esempi utili per scendere in campo nel significato di 'impegnarsi nella lotta politica, in un'impresa di carattere economico, in una disputa ideologica', i primi due - di Vittorini e Bilenchi - dipendono piuttosto da un'immagine bellica che da una sportiva; qualche incertezza si può avere per il terzo e più recente, tratto da un articolo del quotidiano "La Repubblica" del I5 gennaio I986: "Da ieri pomeriggio il vicesegretario socialista è sceso in campo per tentare l'ultima mediazione sulla presidenza Carniti per la Rai-TV": la sede e l'argomento - giornale e politica - potrebbero spostare la locuzione sul piano sportivo.

Il campo dunque è il luogo dello scontro o del confronto, nel quale del tutto lecitamente anche il politico potrà intervenire; quindi, per dirla con Michele Loporcaro (e con Mario Alinei), solo il prevalere nella nostra società di una determinata sfera iconimica - la motivazione cioè del segno linguistico, motivazione che è radicata nelle circostanze sociali - induce l'immediato pensiero dell'immagine sportiva, quando in altri tempi sarebbe stata la guerra o il duello: la contemporanea centralità sociale dello sport ne determina anche lo sfruttamento metaforico delle specifiche espressioni linguistiche.

Sembra confermare queste considerazioni una rapida indagine nell'archivio storico di uno dei più antichi quotidiani italiani: "La Stampa" di Torino. Le attestazioni di scendere in campo e mettere in campo ritrovate tra il I867 e il I868, nei primi due anni di vita cioè del giornale che allora si chiamava "Gazzetta piemontese", o rimandano direttamente all'ambito militaresco o a questo alludono quando usate con significato figurato; mai, ed è del tutto prevedibile, hanno a che fare con lo sport: "Se domani tutte le potenze europee, decise nel concorde proposito di non turbar l'equilibrio attuale delle cose negassero scendere in campo, metterebbero il Governo francese nella situazione di quell'artista da teatro che presentandosi sulla scena volesse recitar la commedia da sé solo" (I5.08.i868, p. 3). Per rintracciare le due locuzioni nel significato sportivo - pur ammettendo un'indagine a campione e non esaustiva - bisognerà attendere gli anni Dieci del Novecento: "Le due squadre scesero in campo nella seguente formazione" (I5.04.I9I2, p. 6), "La squadra cosi composta è la migliore che il Zurich possa mettere in campo, ed è tale l'undici che nella corrente stagione non è stato ancora battuto una volta" (I4.04.I9II, p. 2).

Se può essere significativa la retrodatazione di circa un quarantennio rispetto al GDLI per scendere in campo, va però detto che si tratta di attestazioni del tutto isolate: per la prima metà del secolo raramente si incontrano le due locuzioni nel significato sportivo. La motivazione delle due espressioni rimarrà ancora legata, almeno fino alla fine degli anni Cinquanta del secolo scorso, all'ambito bellico, militaresco, e solo a partire dagli anni Ottanta e Novanta a essa si sostituirà pienamente una motivazione legata allo sport, divenuto aspetto centrale della società.

Ma, per concludere, un ultimo esempio dalla "Stampa" ottocentesca, dal quale si scopre che anche i linguisti possono scendere in campo; così si leggeva nell'edizione del 6 dicembre i868, p. I:

Nel recente rinfocolarsi della eterna quistione intorno a questo nostro linguaggio, rinfocolarsi cui diede origine la oramai famosa scrittura dell'illustre Alessandro Manzoni che fu la relazione d'una Commissione a ciò nominata dal presente ministro della istruzione pubblica; era più che un diritto, era un dovere nel signor Fanfani di scendere in campo e recare in più precisi e concreti termini il complesso e il risultamento delle sue opinioni e de' suoi studi... 
Per approfondimenti:

- G. Antonelli, Sullitaliano dei politici nella seconda Repubblica, in S. Vanvolsem et alii, Italiano oltre frontiera, Firenze, Cesati, 2000, vol. I, pp. 2II-234.

- C. Bascetta, Il linguaggio sportivo contemporaneo, Firenze, Sansoni, I962.

- G.L. Beccaria (a cura di), I linguaggi settoriali in Italia, Milano, Bompiani, I973.

- G.L. Beccaria, Per difesa e per amore. La lingua italiana oggi, Milano, Garzanti, 2006.

- M. Dardano, La lingua dei media, in V. Castronovo, N. Tranfaglia (a cura di), La stampa italiana nell'etá della TV. Dagli anni Settanta a oggi, Roma-Bari, Laterza, 2002, pp. 243-285.

- M. V. Dell'Anna, P. Lala, Mi consenta un girotondo. Lingua e lessico nella Seconda Repubblica, Galatina, Congedo, 2004.

- R. Gualdo, M.V. Dell'Anna, La faconda repubblica. La lingua della politica in Italia (1992-2004), Lecce, Manni, 2004.

- M. Loporcaro, Cattive notizie. La retorica senza lumi dei mass media italiani, Milano, Feltrinelli, 2005 .

- D. Proietti (a cura di), Ludus la lingua in gioco. Linguistica italiana e sport (1939-1992), Roma, Pellicani,

- R. Vetrugno, C. De Santis, C. Panzieri, F. Della Corte (a cura di), Litaliano al voto, Firenze, Accademia della Crusca, 2008.

\section{Cita come:}

Mario Piotti, Scendere in campo /mettere in campo , "Italiano digitale", 2014, XII, 2020/1 (gennaiomarzo)

DOI: $10.35948 / 2532-9006 / 2020.3297$

\section{Copyright 2014 Accademia della Crusca}

Pubblicato con licenza creative commons CC BY-NC-ND 